\title{
EDITORIAL Responses of pediatric palliative care to the COVID-19 pandemic in China
}

\author{
Pediatric Research (2021) 89:1320-1321; https://doi.org/10.1038/s41390- \\ 020-01137-3
}

The widespread outbreak of the coronavirus disease 2019 (COVID19) has been a significant global concern. As of July 23, 2020, $15,012,731$ COVID-19 cases have been confirmed, resulting in 619,150 deaths globally. ${ }^{1}$ A systematic review reported that children have accounted for $1-5 \%$ of diagnosed cases. ${ }^{2} \mathrm{~A}$ basic principle of pediatric palliative care (PPC) is to improve the quality of life for seriously ill children and their families, whether they are suffering from severe COVID-19 or another disease. Providing effective palliative care is especially vital but difficult in the context of a pandemic. ${ }^{3}$ Palliative care has never been so important; however, palliative care is underemphasized in the World Health Organization's essential health services' guidance. ${ }^{3}$ This paper aims to describe the impacts of the COVID-19 pandemic on PPC in China and the response of PPC to the pandemic based on the experience of a tertiary children's hospital in Beijing, China.

\section{IMPACTS OF THE COVID-19 PANDEMIC ON PPC}

The accessibility of PPC has been seriously impacted by the COVID-19 pandemic in terms of palliative care inpatient and outpatient services as well as home visits. During the pandemic, the palliative care ward has set strict admission standards. Children and their family members have had to undergo strict screening procedures, including symptom screening, COVID-19 contact history assessments, nucleic acid tests, and chest computed tomographic scans. Children with qualified screening results after 2 weeks of isolation could be admitted to the ward. The admission standards for nonlocal children were even stricter than for those from local areas. Infection prevention and control (IPC) measures during the COVID-19 pandemic resulted in delayed transfer to the palliative care ward, and some children could not even be admitted to the ward.

After entering the ward, the families still faced the situation of isolation from the children. Compared with adult patients, children and their families had a stronger demand for company. However, under the restriction of the quarantine policy during the pandemic, most palliative care wards have allowed only one family member to accompany the child, which was unacceptable to some families. It was extremely difficult to find a balance between epidemic control and the need for visitation during the pandemic. Furthermore, due to the strict implementation of IPC measures, the PPC team had to stop home visits and limit inpatient services, and parents had difficulty obtaining outpatient consultation and necessary medications.

The shortage of services in PPC negatively impacted the quality of life of both seriously ill children, especially children with cancer, and their families. Children with cancer had more severe symptoms than those with other terminal illnesses. They generally suffer severe pain at the end of life and need professional pain management. However, it was difficult for those children to obtain timely and effective symptom management during the pandemic.
Poor symptom management could significantly affect the quality of life of children, thereby causing psychological distress for the families. In addition, the pandemic made resource-scarce psychological and spiritual support more difficult to obtain for children and their families.

The greatest challenge facing the PPC team was the ability to adjust their work patterns to ensure service quality in the context of pandemic prevention. In addition, the PPC team had to face insufficient staffing levels, the risk of becoming infected or infecting others when providing care, and heavy demands from desperate families, which could negatively affect the well-being of palliative care providers and patient outcomes. ${ }^{4}$

\section{RESPONSE OF PPC TO THE COVID-19 PANDEMIC}

To maintain a safe, healthy working environment and guarantee service quality during the pandemic, the PPC team adjusted their work patterns. To reduce the risk of infection, only some core team members, such as nurses and physicians, were allowed to enter the palliative care ward to provide services. The supply of personal protection equipment, sanitary, and cleaning products was guaranteed. In addition, prolonged work shifts with continuous services were implemented. To meet the needs of families, some palliative care wards allowed one to two family members to accompany patients, prohibiting family caregivers from exiting or alternating caregivers. Families' necessities were purchased with the help of the PPC team. The PPC team provided timely information about the children to the relatives who could not visit the ward by texting or calling them. For family members of dying children, if they had no suspected contact with patients with confirmed COVID-19 and if their nucleic acid testing was negative, they were allowed to visit and accompany their children so that their children could enjoy terminal visitation.

As a supplement, online services such as virtual visits for homeand community-based patients were implemented to provide symptom management, psychosocial care, and bereavement support. The PPC team provided online services through the "online ward," an online platform for medical record management and inquiry as well as patient follow-up. During the pandemic, the PPC team admitted and managed patients through the "online ward." The PPC team obtained the demographic and medical information and needs of the child in the "online ward" and offered support accordingly. Although online services were widely used by PPC teams during the pandemic, PPC providers found the provision of virtual care challenging and expressed concern about their ability to provide the same level of quality care in the absence of face-to-face encounters with patients. Physicians in particular were worried about their ability to manage symptoms based solely on patient reports, without corroborating evidence from physical examinations and laboratory tests.

\section{PPC WORK PATTERNS IN THE POSTPANDEMIC PERIOD}

As the epidemic is nearly controlled in China, hospitals are preparing to fully advance work resumption. However, as the 
pandemic continues its global spread, China is under rising pressure to guard against imported COVID-19 cases from abroad and local sporadic cases. Therefore, healthcare systems are making arrangements to fully advance work resumption while adhering to regular IPC measures. Hospitals are required to undertake responsibilities to strengthen safety management while organizing work resumption.

PPC teams need to be flexible and rapidly adjust their work patterns in the face of changing needs and emergency response levels, especially in metropolitan areas where the pandemic situation is constantly changing. PPC teams must guarantee a safe environment and quality service through the combined utilization of online and onsite services. PPC teams gradually increase the frequency of onsite services with strengthened personal protection during physical contacts. The screening procedure and regulations for visitation to the palliative care ward are continuously adjusted in accordance with guidance from the Centers for Disease Control and Prevention. In addition, PPC teams have replaced in-person activities, such as meetings, trainings, and consultations, with online versions of such activities.

\section{ROLE OF PPC IN THE COVID-19 PANDEMIC}

The principles of PPC could be applied to children with COVID-19. Although the condition of most children with COVID-19 was not severe, they still suffered from fear, uncertainty, and physical and social isolation. Separation from family members could increase the risk of psychiatric disorders in children with COVID-19. PPC teams can address the emotional, social, and spiritual needs of children with COVID-19 by listening to children's questions, such as "How did I get it?," "Is this going to get worse?," or even "Will I die from this?" Answering honest responses appropriate for the child's developmental age can reduce their anxiety, confusion, and misperceptions. Weaver et al. provided the method of adults sharing certain grounding values in communication about COVID19 with children. ${ }^{5}$ Death education and grief counseling could play special roles in the dying process and bereavement of seriously ill children with COVID-19 and their parents to maintain comfort and relieve fear of death and grief. PPC addresses the needs of families of patients with COVID-19. In the face of COVID19 , the whole family will fall into great panic, and a hospital's "novisitor policy" leads them to suffer from separation from each other. PPC teams can provide family members with online psychological support, COVID-19-related knowledge, and methods of channeling children's negative emotions and answering children's questions.

The compassion and caring that palliative care providers offer every day inspire another role of PPC in the time of COVID-19, which is to maintain the element of humanity in the care of the sick children and their families as well as stressed healthcare providers by offering sympathy and compassion. However, to achieve these roles in a global pandemic, we need universal PPC.

\section{ACKNOWLEDGEMENTS}

This work was supported by the National Natural Science Foundation of China [grant number 81803102] and the Chinese Society of Medical Education of Chinese Medical Association and the Professional Committee for Medical Education of China Association of Higher Education [grant number 2018B-N17003].

\section{AUTHOR CONTRIBUTIONS}

Q.G. contributed to the conceptualization and design of the study. All the authors drafted the manuscript, with major contributions from S.C. X.Z. and Q.G. critically revised the manuscript.

\section{ADDITIONAL INFORMATION}

Competing interests: The authors declare no competing interests.

Informed consent: Informed consent was not required, as this study did not involve human subjects.

Publisher's note Springer Nature remains neutral with regard to jurisdictional claims in published maps and institutional affiliations.

Xuan Zhou', Siyu $\mathrm{Cai}^{2}$ and Qiaohong Guo ${ }^{3}$

${ }^{1}$ Beijing Key Laboratory of Pediatric Hematology Oncology; National Key Discipline of Pediatrics (Capital Medical University); Key Laboratory of Major Diseases in Children, Ministry of Education; Hematology Oncology Center, Beijing Children's Hospital, Capital Medical University, National Center for Children's Health, Beijing, China; ${ }^{2}$ Center for Clinical Epidemiology and Evidence-based Medicine, Beijing Children's Hospital, Capital Medical University, National Center for Children's Health, Beijing, China and ${ }^{3}$ School of Nursing, Capital Medical University, Beijing, China These authors contributed equally: Xuan Zhou, Siyu Cai. Correspondence: Qiaohong Guo (guoqiaohong07@163.com)

\section{REFERENCES}

1. World Health Organization. Coronavirus disease 2019 (COVID-19) situation report 185. https://www.who.int/docs/default-source/coronaviruse/situation-reports/ 20200723-covid-19-sitrep-185.pdf?sfvrsn=9395b7bf (2020). Accessed 23 Jul 2020.

2. Ludvigsson, J. F. Systematic review of COVID-19 in children showsmilder cases and a better prognosis than adults. Acta Paediatr. 109, 1088-1095 (2020).

3. The Lancet. Palliative care and the COVID-19 pandemic. Lancet 395, 1168 (2020).

4. Radbruch, L., Knaul, F. M., de Lima, L., de Joncheere, C. \& Bhadelia, A. The key role of palliative care in response to the COVID-19 tsunami of suffering. Lancet https:// doi.org/10.1016/S0140-6736(20)30964-8 (2020).

5. Weaver, M. S. \& Wiener, L. Applying palliative care principles to communicate with children about COVID-19. J. Pain Symptom Manage. https://doi.org/10.1016/j. jpainsymman.2020.03.020 (2020). 